\title{
Transcriptome Assessment of Erythema Migrans Skin Lesions in Patients With Early Lyme Disease Reveals Predominant Interferon Signaling
}

\author{
Adriana Marques, ${ }^{1}$ Ira Schwartz, ${ }^{2}$ Gary P. Wormser, ${ }^{3}$ Yanmei Wang, ${ }^{4}$ Ronald L. Hornung, ${ }^{4}$ Cumhur Y. Demirkale, ${ }^{5}$ Peter J. Munson, ${ }^{5}$ \\ Siu-Ping Turk, ${ }^{1}$ Carla Williams, ${ }^{4}$ Chyi-Chia Richard Lee, ${ }^{6}$ Jun Yang, ${ }^{7}$ and Mary M. Petzke ${ }^{2}$ \\ ${ }^{1}$ Laboratory of Clinical Infectious Diseases, National Institute of Allergy and Infectious Diseases, National Institutes of Health, Bethesda, Maryland; ${ }^{2}$ Department of Microbiology \\ and Immunology and ${ }^{3}$ Division of Infectious Diseases, Department of Medicine, New York Medical College, Valhalla; ${ }^{4}$ Clinical Services Program, Leidos Biomedical Research, \\ Inc., Frederick National Laboratory for Cancer Research, Maryland; ${ }^{5}$ Mathematical and Statistical Computing Laboratory, Center for Information Technology, National Institutes \\ of Health, Bethesda, Maryland; ' ${ }^{6}$ Laboratory of Pathology, Center for Cancer Research, National Cancer Institute, National Institutes of Health, Bethesda, Maryland; ${ }^{7}$ Laboratory of \\ Human Retrovirology and Immunoinformatics, Leidos Biomedical Research, Inc. Frederick National Laboratory for Cancer Research, Maryland
}

Background. The most common clinical manifestation of early Lyme disease is the erythema migrans (EM) skin lesion that develops at the tick bite site typically between 7 and 14 days after infection with Borreliella burgdorferi. The host-pathogen interactions that occur in the skin may have a critical role in determining outcome of infection.

Methods. Gene arrays were used to characterize the global transcriptional alterations in skin biopsy samples of EM lesions from untreated adult patients with Lyme disease in comparison to controls.

Results. The transcriptional pattern in EM biopsies consisted of 254 differentially regulated genes ( 180 induced and 74 repressed) characterized by the induction of chemokines, cytokines, Toll-like receptors, antimicrobial peptides, monocytoid cell activation markers, and numerous genes annotated as interferon (IFN)-inducible. The IFN-inducible genes included 3 transcripts involved in tryptophan catabolism (IDO1, KMO, KYNU) that play a pivotal role in immune evasion by certain other microbial pathogens by driving the differentiation of regulatory $\mathrm{T}$ cells.

Conclusions. This is the first study to globally assess the human skin transcriptional response during early Lyme disease. Borreliella burgdorferi elicits a predominant IFN signature in the EM lesion, suggesting a potential mechanism for spirochetal dissemination via IDO1-mediated localized immunosuppression.

Keywords. skin; interferon; Treg; Lyme disease.

Lyme disease, the most common tick-borne disease in the United States and Europe, is caused by Borreliella (Borrelia) burgdorferi [1]. Borreliella burgdorferi is transmitted during the feeding of ticks of the Ixodes ricinus complex. Infection initiates when the bacterium enters the skin at the bite site, multiplying locally and causing a centrifugally spreading annular rash called erythema migrans (EM). Erythema migrans typically develops 7 to 14 days after the bite and results from the migration of spirochetes in an outward direction from the inoculation site. If untreated, hematogenous dissemination of spirochetes may occur, and patients can present with additional skin lesions and/ or neurologic, cardiac, and rheumatologic manifestations [2].

Received 18 July 2017; editorial decision 20 October 2017; accepted 24 October 2017; published online November 1, 2017

Presented in part: 113th Annual General Meeting of the American Society for Microbiology, Denver, CO, May 18-21, 2013; 13th International Conference on Lyme Borreliosis and Other Tick-Borne Diseases, Boston, MA, August 18-21, 2013.

Correspondence: M. M. Petzke, PhD, New York Medical College, Basic Sciences Building, Room 306, 15 Dana Road, Valhalla, NY 10595 (mary_petzke@nymc.edu).

The Journal of Infectious Diseases ${ }^{\circledR} \quad$ 2018;217:158-67

(C) The Author(s) 2017. Published by Oxford University Press for the Infectious Diseases Society of America. All rights reserved. For permissions, e-mail: journals.permissions@oup.com. DOI: 10.1093/infdis/jix563
The first steps of the immune response against $B$ burgdorferi occur in the skin, and this initial interaction between host and pathogen is likely to play a pivotal role in determining the outcome of infection [3]. Histological examination of EM lesions reveals patchy perivascular infiltrates composed mostly of $\mathrm{T}$ lymphocytes, with lesser numbers of histiocytes and B cells [3]. Erythema migrans lesions express messenger ribonucleic acid (RNA) for both inflammatory and anti-inflammatory cytokines, particularly interferon (IFN)- $\gamma$ and interleukin (IL)-10, but also tumor necrosis factor (TNF) $\alpha$, IL-1 $\beta$, and IL-6 [4]. In a study examining fluid from blisters raised over EM lesions, the aspirates were enriched for $\mathrm{T}$ cells, monocytes/macrophages, and dendritic cells (DCs); IL- 6 and IFN- $\gamma$ were the predominant cytokines [5].

In this study, we used gene array analysis to globally assess the human response associated with $B$ burgdorferi infection in EM skin lesions in adult patients with untreated early Lyme disease. Our results show that many different mechanisms are involved in the host response against $B$ burgdorferi in the skin, some well recognized, others representing novel observations, suggesting opportunities for future research. 
METHODS

Ethics Statement

Individuals providing written informed consent were enrolled in studies approved by the institutional review boards at the National Institute of Allergy and Infectious Diseases (National Institutes of Health [NIH]; NCT00132327) or at New York Medical College (NYMC).

\section{Skin Biopsy Collection and Characterization}

Skin specimens from 18 EM patients [6] and 11 healthy volunteers (HV) were obtained by punch biopsy (2-4 $\mathrm{mm}$ ), after local anesthesia with $2 \%$ lidocaine with epinephrine. Specimens were obtained from the advancing border of EM lesions before initiation of antibiotic therapy. Healthy volunteers had no history or serologic evidence of Lyme disease. Surgical control (SC) skin samples were obtained from specimens of 16 anonymous patients undergoing surgery for other purposes. All skin specimens were stored at $-80^{\circ} \mathrm{C}$ in RNAlater solution (Ambion) before RNA isolation. Cultivation of $B$ burgdorferi from skin and blood samples was performed as described previously $[7,8]$.

\section{Microarray Analysis}

Total RNA was extracted from skin biopsies using a modified RNeasy Fibrous Tissue Mini kit (QIAGEN, Germantown, $\mathrm{MD})$. Ribonucleic acid was quantitated and qualified with Nanodrop 1000 and Agilent Bioanalyzer RNA Nano 6000 chip. Complementary RNA (cRNA) synthesis, labeling, and hybridization to the GeneChip Human Genome U133A 2.0 or U133Plus 2.0 Array (Affymetrix, St. Clara, CA) were performed according to the manufacturer's protocol. Microarrays were scanned at $570 \mathrm{~nm}$ using Affymetrix Genechip Scanner 3000 7G. The resulting CEL files were analyzed using Affymetrix Expression Console and the MAS5 algorithm. Resulting Signal Intensity values and Present-Absent calls for each of the probesets were processed in JMP statistical package (SAS, Cary, NC) using the Mathematical and Statistical Computing Laboratory (MSCL) Analyst's Toolbox, written by and freely available (http://abs.cit.nih.gov/MSCLtoolbox/) from one of the authors (P.J.M.). The 22277 probe sets in common between HG-U133A 2.0 and HG-U133 plus 2.0 chips were used for further analysis. The quantile-normalizing, variance-stabilizing "S10" transform was applied to the Signal Intensity data. Principle component analysis was performed to identify global variance and potential outliers in the data. One-way analysis of variance (ANOVA) was performed on each of the low-order principle components to test whether the components can be explained by either block effect or group effect (EM vs control). Functional enrichment analysis was performed using DAVID (https://david.ncifcrf. gov/home.jsp) [9] and Ingenuity Pathway Analysis (Ingenuity Systems, www.ingenuity.com).
Quantitative Real-Time Reverse-Transcription Polymerase Chain Reaction Transcriptional expression was measured by reverse-transcription polymerase chain reaction (RT-PCR) using the remaining cRNA from $10 \mathrm{HV}$ and 10 EM biopsies (5 NIH, 5 NYMC) that had been included in the microarray analysis. The list of probe and primer sets is available as Supplementary Methods. A $\Delta \Delta \mathrm{C}_{\mathrm{T}}$ method was used to calculate the differential expression of each $G A P D H$-normalized gene in EM relative to healthy controls (HCs).

\section{Immunohistochemical Staining}

Skin biopsies were fixed in 10\% neutral buffered formalin and embedded in paraffin blocks. Tissue sections $(5 \mu \mathrm{m})$ were prepared and placed on poly-L-lysine-coated glass slides. After antigen retrieval, tissue sections were incubated with primary antibodies (see Supplementary Methods). Staining was performed on the Roche Ventana Medical Systems BenchMark ULTRA automated immunohistochemistry (IHC) platform using the ultraView Universal DAB Detection Kit (Ventana Medical Systems), after standard protocols of the histology section of the Laboratory of Pathology at NIH. Three areas were visually scored by a dermatopathologist (C.-C.R.L.), and the cell counts were averaged to give the number of cells per high-powered field, defined as $4.5-\mathrm{mm}^{2}$ field of view at $\times 40$ objective magnification. The superficial (papillary) and deep (reticular) dermis were scored separately.

\section{Statistics}

Statistical significance of differences between the mean RT-PCR fold-change values of experimental groups was determined using a 2-tailed, unpaired $t$ test, assuming either equal or unequal variance, as determined by Fisher's F-test (Microsoft Excel, Microsoft, Inc.). Correlation between fold-change values obtained either by microarray or by RT-PCR was assessed using linear regression analysis (Excel). The IHC data were analyzed by the nonparametric Mann-Whitney test using GraphPad Prism, version 7.0b (GraphPad Software, San Diego, CA). Twotailed $P$ values of less than .05 were considered significant.

\section{RESULTS}

\section{Patient Characteristics}

Eighteen subjects with EM were evaluated (Table 1). These included 8 subjects enrolled at the NIH Clinical Center in Bethesda, Maryland, and 10 subjects enrolled at the Lyme Disease Diagnostic Center at NYMC. Subjects at the NIH site were enrolled consecutively without regard to age, gender, or number of EM skin lesions. The NYMC samples were selected from a repository of stored biopsies from patients known to have had a positive blood culture for B burgdorferi, and therefore all are classified as having disseminated infection. There were 15 females and 3 males. The majority of Lyme subjects 
Table 1. Clinical Characteristics of Lyme Disease Subjects

\begin{tabular}{|c|c|}
\hline Total & $\mathbf{n}=18$ \\
\hline \multicolumn{2}{|l|}{ Gender } \\
\hline Male & $3(17 \%)$ \\
\hline Female & $15(83 \%)$ \\
\hline \multicolumn{2}{|l|}{ Age } \\
\hline$<60$ years & $12(67 \%)$ \\
\hline$\geq 60$ years & $6(33 \%)$ \\
\hline \multicolumn{2}{|l|}{ Erythema migrans (EM) rash } \\
\hline Size, $\mathrm{cm}^{2}$, median (range) & $84.5(34-352)$ \\
\hline Duration, days, median (range) & $4(1-15)$ \\
\hline Multiple EM (MEM) & $6(33 \%)$ \\
\hline \multicolumn{2}{|c|}{ Seropositive, B burgdorferi enzyme-linked immunosorbent assay } \\
\hline Initial visit & $11(61 \%)$ \\
\hline 1-month return visit ${ }^{a}$ & 17 of $17(100 \%)$ \\
\hline \multicolumn{2}{|l|}{ Skin culture for $B$ burgdorferi } \\
\hline Positive & $13(72 \%)$ \\
\hline Negative & $1(6 \%)$ \\
\hline Contaminated & $2(11 \%)$ \\
\hline Not done & $2(11 \%)$ \\
\hline \multicolumn{2}{|l|}{ Blood culture for B burgdorferi } \\
\hline Positive & $13(72 \%)$ \\
\hline Negative & $4(22 \%)$ \\
\hline Not done & $1(6 \%)$ \\
\hline \multicolumn{2}{|l|}{ Disseminated infection } \\
\hline MEM and/or positive blood culture & $14(78 \%)$ \\
\hline \multicolumn{2}{|l|}{ Systemic symptoms } \\
\hline Present & $14(78 \%)$ \\
\hline Number, median (range) & $2(0-8)$ \\
\hline Fatigue & $9(50 \%)$ \\
\hline Headache & $7(39 \%)$ \\
\hline Arthralgias & $7(39 \%)$ \\
\hline Fever & $6(33 \%)$ \\
\hline Local adenopathy & $2(11 \%)$ \\
\hline
\end{tabular}

(70\%) were between 40 and 69 years of age. The mean age of the 11 HCs was 36 years; and 7 were female. All 16 SCs were female.

Five patients recalled a tick bite at the site of the EM skin lesion. Erythema migrans lesions varied in size, with a median area of $84.5 \mathrm{~cm}^{2}$ (range, 34 to $352 \mathrm{~cm}^{2}$ ) (for the 6 patients with multiple EM skin lesions, the size is that of the largest lesion). The median duration of EM before the lesion was biopsied was 4 days (range, 1 to 15 days). Lyme subjects were questioned regarding the presence of appetite loss, arthralgia, cough, dizziness, fatigue, fever, headache, myalgia, nausea and vomiting, paresthesias, stiff neck, and concentration or memory difficulties. Fourteen Lyme subjects had at least 1 symptom at evaluation, with a median of 2 symptoms present. The most common symptoms were fatigue (9 of 18), headache (7 of 18), and arthralgias (7 of 18).

Culture of the skin biopsy was positive in 13 patients, negative in 1 individual, contaminated in 2 individuals, and not performed in 2 individuals. Blood culture for B burgdorferi was performed for 7 patients at the NIH site; the test was positive for
3 patients. Blood culture was not done in 1 patient with multiple EM lesions. A B burgdorferi enzyme-linked immunosorbent assay ([ELISA] C6 ELISA at the NIH, and whole cell sonicate ELISA at NYMC) was positive at the initial visit in 11 of the 18 Lyme subjects, and it was positive at the 1-month visit in all 17 of the Lyme subjects tested. Overall, 14 Lyme subjects were classified as having disseminated infection by either presenting with multiple EM lesions and/or having a positive blood culture for B burgdorferi.

\section{Gene Expression Profiles of Erythema Migrans Lesions}

To characterize the host response to $B$ burgdorferi infection in EM lesions, we compared gene expression in EM skin biopsies from 18 Lyme subjects with skin samples from 27 controls. The study samples were processed in 2 batches. Batch 1 included 31 samples (16 skin specimens from SC, 5 NIH EM biopsies, and 10 NYMC EM biopsies). Batch 2 included 14 samples (11 skin biopsies from HV and 3 NIH EM biopsies). A principal components analysis was run on all 45 samples. Figure 1 shows that the first principal component explains $23.7 \%$ of the variation in the data, and it clearly separates controls from EM. The second principal component separated the 2 groups of controls (SC and HV). A separate analysis (data not shown) demonstrated that the batch effect explained more of the variation in the second principal component than did the differences between the collection methodologies (surgical vs punch biopsy) used for the 2 control groups. Therefore, batch was included as a factor in the subsequent analysis. A one-way, 2-level blocked ANOVA was performed, using batch as a blocking factor and the group (EM or controls [SC and HV combined]) as the factor of interest. Probe sets that were defined as significantly differentially expressed met a false discovery rate $\leq 10 \%$, had a minimum 3 -fold change in expression between EM and controls, and were present in at least $50 \%$ of the samples in 1 of the 2 groups. Using these criteria, 327 probe sets (254 genes) were differentially expressed; 180 genes were upregulated and 74 genes downregulated in EM lesions when compared with controls. Complete lists of all significantly differentially regulated genes are provided in Supplementary Tables 1 and 2; a selected list of induced genes with fold change values is presented in Table 2.

\section{Validation of Microarray Results}

Twenty genes were selected from the microarray data set and validated by real-time RT-PCR. Although the absolute values obtained by gene array and RT-PCR varied, an excellent linear correlation was observed between the 2 data sets $\left(r^{2}=0.87\right)$ (Supplementary Table 3 and Supplementary Figure).

\section{Genes Related to Different Branches of the Immune System Are Induced in Erythema Migrans Lesions}

Gene ontology enrichment analysis was performed using the DAVID Gene Functional Classification Tool [9], with an EASE Score Threshold (a modified Fisher exact $P$ value of .001). The biological 


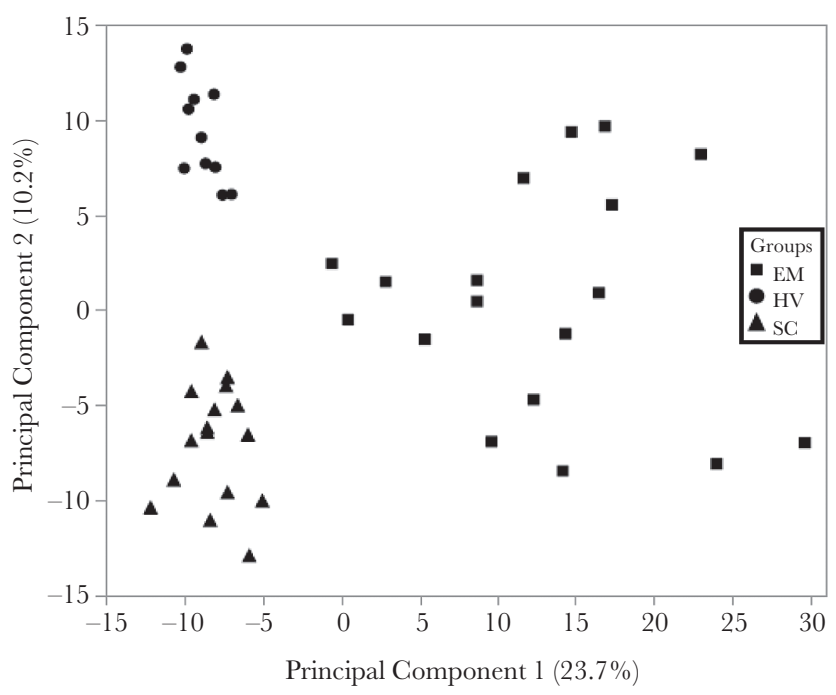

Figure 1. Principal components analysis distinguishes patients with early untreated Lyme disease from healthy donors. Abbreviations: EM, skin punch biopsies from erythema migrans lesion; HV, skin punch biopsies from healthy volunteers; SC, breast skin tissue from healthy patients undergoing elective surgery. processes associated with genes that were induced in EM lesions included innate immune response, cell migration and chemotaxis, and defense responses to both bacteria and viruses (Supplementary Table 4). Biological processes significantly represented among repressed genes in EM consisted of functions involved in tissue development and remodeling (Supplementary Table 4).

Ingenuity Pathway Analysis (www.ingenuity.com) was used to help identify relationships, mechanisms, functions, and pathways relevant to a dataset. The top 10 canonical pathways, upstream regulators, and disease and biological functions regulated in EM are shown in Figure 2. Interferon signaling was the top canonical pathway $\left(P=3.15 \times 10^{-18}\right)$ that showed significant activation, followed by pattern recognition receptors in recognition of bacteria and viruses, and DC maturation. The top 5 upstream regulators included IFN- $\gamma$, lipopolysaccharide, IFNa, Poly rI:rC-RNA, and TNF.

\section{Interferon Signaling Is Predominant Transcriptional Profile in Erythema Migrans}

We used Interferome [10] (http://interferome.its.monash.edu. $\mathrm{au} /$ interferome/home.jspx) to analyze the gene list for IFN

Table 2. Selected Transcripts Induced in EM Skin Biopsies Relative to Controls (HV and SC)

\begin{tabular}{|c|c|c|c|c|}
\hline Gene Symbol & Gene Name & Entrez Gene & Fold Change ${ }^{a}$ & Cluster ID \\
\hline GBP1 & Guanylate binding protein 1, interferon-inducible, $67 \mathrm{kDa}$ & 2633 & 22.76 & 1 \\
\hline IFI44L & Interferon-induced protein 44-like & 10964 & 14.45 & 1 \\
\hline CCL8 & Chemokine (C-C motif) ligand 8 & 6355 & 12.36 & 1 \\
\hline IFIT3 & Interferon-induced protein with tetratricopeptide repeats 3 & 3437 & 10.14 & 1 \\
\hline OAS2 & 2 '-5'-oligoadenylate synthetase $2,69 / 71 \mathrm{kDa}$ & 4939 & 8.87 & 1 \\
\hline MX1 & Myxovirus (influenza virus) resistance 1, interferon-inducible protein p78 (mouse) & 4599 & 8.32 & 1 \\
\hline STAT1 & Signal transducer and activator of transcription 1,91kDa & 6772 & 6.96 & 1 \\
\hline JAK2 & Janus kinase 2 & 3717 & 3.59 & 1 \\
\hline IRF7 & Interferon regulatory factor 7 & 3665 & 3.36 & 1 \\
\hline IL1B & Interleukin 1, beta & 3553 & 3.17 & 1 \\
\hline S100A8 & S100 calcium binding protein A8 & 6279 & 18.56 & 2 \\
\hline S100A9 & S100 calcium binding protein A9 & 6280 & 10.87 & 2 \\
\hline S100A7 & S100 calcium binding protein A7 & 6278 & 5.14 & 2 \\
\hline CXCL10 & Chemokine (C-X-C motif) ligand 10 & 3627 & 357.27 & 3 \\
\hline CXCL9 & Chemokine (C-X-C motif) ligand 9 & 4283 & 134.45 & 3 \\
\hline CXCL11 & Chemokine (C-X-C motif) ligand 11 & 6373 & 95.54 & 3 \\
\hline FCGR1B & Fc fragment of IgG, high affinity Ib, receptor (CD64) & 2210 & 14.97 & 4 \\
\hline LILRB2 & Leukocyte immunoglobulin-like receptor, subfamily B, member 2 & 10288 & 14.95 & 4 \\
\hline FCGR1A & Fc fragment of IgG, high affinity la, Ic receptor (CD64) & 100132417 & 12.26 & 4 \\
\hline IDO1 & Indoleamine 2,3-dioxygenase 1 & 3620 & 9.05 & 4 \\
\hline CCL19 & Chemokine (C-C motif) ligand 19 & 6363 & 8.92 & 4 \\
\hline CXCL13 & Chemokine (C-X-C motif) ligand 13 & 10563 & 5.65 & 4 \\
\hline TLR2 & Toll-like receptor 2 & 7097 & 4.33 & 4 \\
\hline KYNU & Kynureninase (L-kynurenine hydrolase) & 8942 & 4.17 & 4 \\
\hline CCR5 & Chemokine (C-C motif) receptor 5 & 1234 & 3.75 & 4 \\
\hline $\mathrm{KMO}$ & Kynurenine 3-monooxygenase (kynurenine 3-hydroxylase) & 8564 & 3.43 & 4 \\
\hline IL15 & Interleukin 15 & 3600 & 3.36 & 4 \\
\hline TLR1 & Toll-like receptor 1 & 7096 & 3.25 & 4 \\
\hline IFNG & Interferon gamma & 3458 & 3.2 & 4 \\
\hline
\end{tabular}

Abbreviations: EM, erythema migrans; HV, healthy volunteers; ID, identification; SC, surgical control.

${ }^{a}$ Transcripts within each cluster are organized by fold change. For genes detected by multiple probe sets, the fold-change values of all probe sets were averaged. 
regulation. Analysis of the current data set was restricted to human species and with a fold change of $\geq 5$. Of the 254 differentially expressed genes, 109 (43\%) were identified as IFNregulated genes (IRGs). Of these 109 IRGs, 74 genes were regulated by both IFN types I and II: 19 genes by IFN type I alone, and 16 genes by IFN type II only. The IRGs with differential expression in EM lesions included IFNG, IFIT3, OAS1, MX1, IFI35, PSMB8, JAK2, TAP1, IRF1, ISG15, IFIT1, IFI6, STAT1, and IFITM1.

\section{Hierarchical Clustering Analysis}

To investigate the relationships between the differentially expressed genes, the list of probe sets was used for hierarchical clustering to generate a heat map (Figure 3). As with the principal components analysis, EM and controls clustered separately. Upregulated genes in EM separated into 4 clusters: 2 large (Cluster 1 [89 probe sets] and Cluster 4 [81 probe sets]) and 2 small (Cluster 2 [7 probe sets] and Cluster 3 [3 probe sets]). Downregulated genes separated into 2 clusters (Cluster 5 [7 probe sets] and Cluster 6 [67 probe sets]) (Figure 3).

The T-cell chemoattractants CXCL9, CXCL10, and CXCL11, the most highly induced genes in our dataset, comprised Cluster 3. Cluster 2 included genes related to damage-associated molecular patterns or alarmins. Genes in this cluster include S100A8 (myeloid-related protein [MRP] 8, calgranulin A), S100A9 (MRP14, calgranulin B), and S100A7 (psoriasin). Also in this cluster are the genes for type I keratin 16 (KRT16) and type II keratin 6 (KRT6a, KRT6b), which are involved in skin barrier maintenance and innate immunity.

Cluster 1 included the majority (88 of 109) of IRGs. Pathway analysis identified IFN signaling, role of pattern recognition receptors in recognition of bacteria and viruses, and activation of IRF (IFN response factor) by cytosolic pattern recognition receptors as the top canonical pathways. Interferon- $\gamma, \mathrm{IFN}-\alpha 2$, and IRF7 were the top upstream regulators.

In contrast, Cluster 4 contained transcripts primarily related to innate immune cell functions, including phagocytosis, pathogen recognition, and cytokine secretion. Phagosome formation was the top canonical pathway associated with this cluster. Transcripts included the Fc gamma receptors FCGR1A, FCGR1B, and FCGR3A/ FCGR3B; Toll-like receptors TLR1 and TLR2; and B-cell chemoattractants CXCL13 and CCL19. Cluster 4 also contained the gene for IFN- $\gamma$ (IFNG), the sole IFN found to be induced in EM biopsies. Cluster 4 featured 3 genes that encode key enzymes in tryptophan catabolism: IDO1 (indoleamine 2,3-dioxygenase 1; 9.05-fold), kynurenine 3-monooxygenase (KMO; 3.43-fold), and kynureninase (KYNU; 4.17-fold).

Clusters 5 and 6 comprised genes downregulated in EM lesions. Biological functions associated with these clusters included adhesion of connective tissue cells and cell viability, with transforming growth factor beta being the top upstream regulator.

\section{Erythema Migrans Lesions Are Enriched for T Lymphocytes, Including Regulatory T Cells}

Indoleamine 2,3-dioxygenase 1 expression has been shown to induce immune tolerance by promoting the development of regulatory T cells (Tregs) [11]. Therefore, we analyzed the EM inflammatory infiltrate for the presence of Tregs and DCs in 8 EM lesions and compared this with 4 controls (2 HV and 2 SC). Histopathological features of EM lesions showed that the number of cells expressing forkhead box P3 (FOXP3), a marker of Tregs, and CD123, the IL-3 receptor a chain, a marker of
$\mathbf{A}$

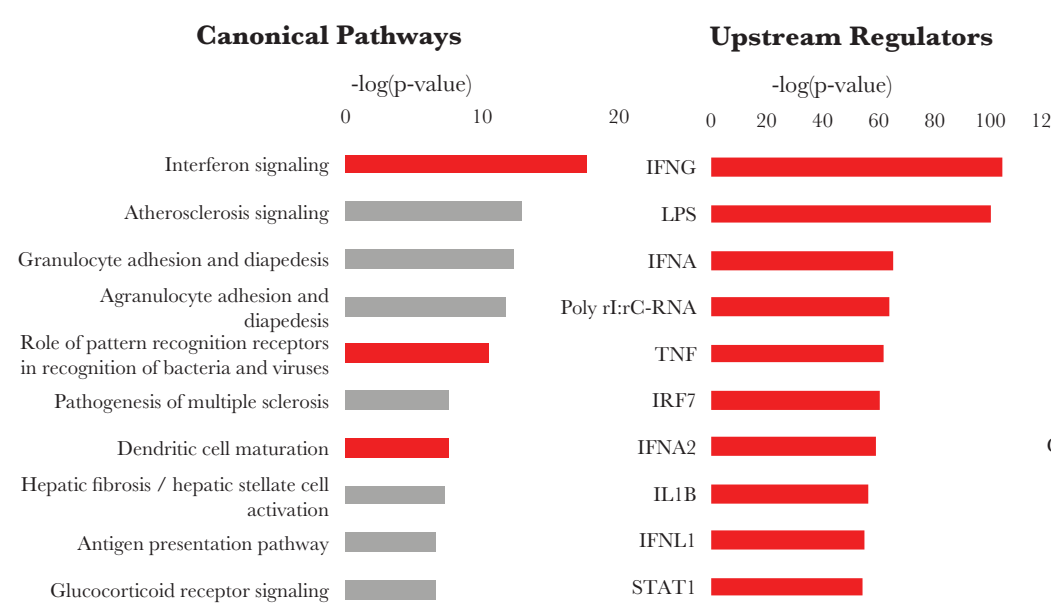

G

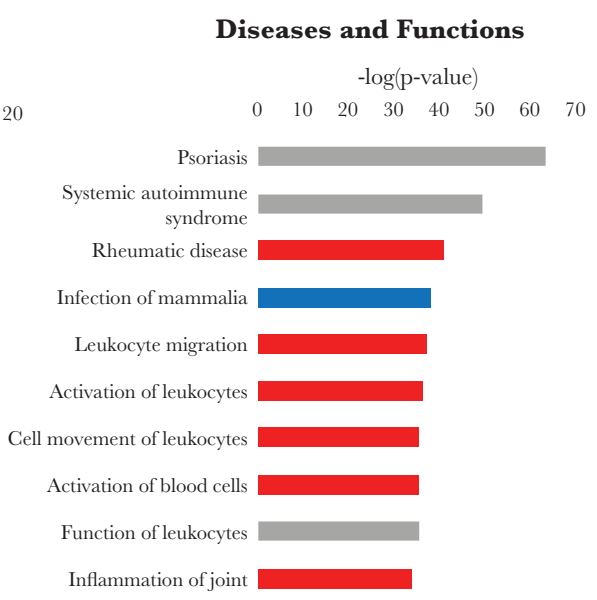

Figure 2. Pathway analysis identifies a predominant interferon signature during early cutaneous Lyme disease. The top $10(A)$ canonical pathways, $(B)$ upstream regulators, and $(C)$ diseases and functions were identified by pathway analysis of probe sets found to be differentially regulated in erythema migrans (EM) biopsies relative to healthy donor skin. Results are ranked by the negative log of the $P$ value of the enrichment score. Bars are colored according to the bias-corrected Z score; activation is shown in red, suppression is shown in blue, and indeterminate directionality is shown in gray. 


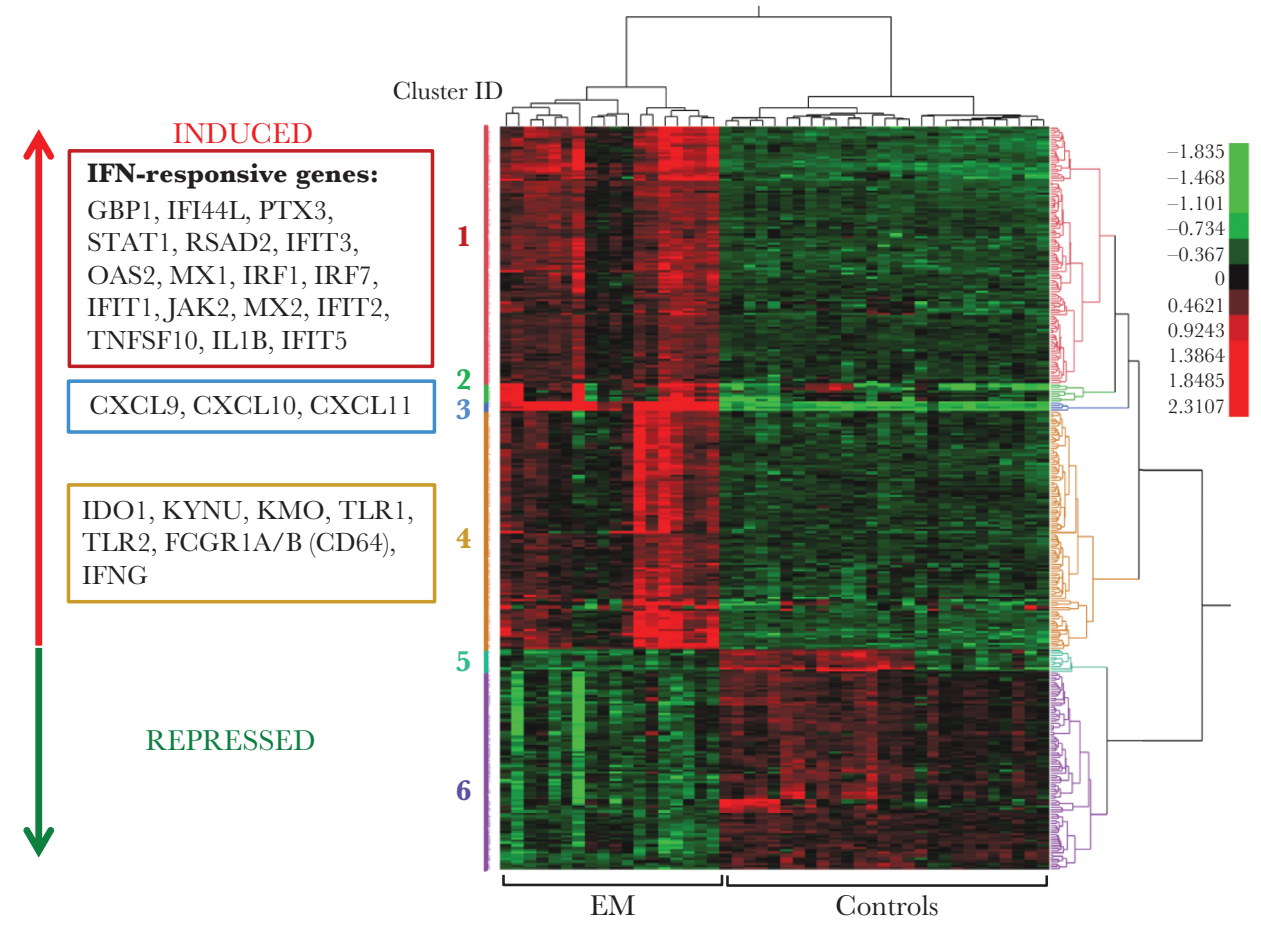

Figure 3. Gene expression profile clearly distinguishes erythema migrans (EM) skin lesions from healthy donor skin. Heat map with the dendrogram resulting from hierarchical clustering performed using 327 probe sets (254 genes; 180 induced, 74 repressed) defined as differentially expressed in skin samples from EM relative to controls (surgical controls and healthy volunteers). The values shown are normalized intensities relative to the mean. Red or green color indicates high or low expression, respectively. The heat map displays 6 distinct clusters, 4 containing induced genes and 2 containing repressed genes. A list of selected significantly induced genes, together with corresponding fold change values, is presented in Table 2. Lists of all differentially regulated probe sets organized by cluster are provided in Supplementary Tables 1 and 2.

plasmacytoid DCs, were significantly increased in EM lesions (Figure 4).

\section{DISCUSSION}

The skin is the portal of entry for $B$ burgdorferi and a critical interface between the spirochete and its human host. However, few studies have interrogated the host response in the EM lesion, and prior studies focused on a limited number of genes and proteins $[4,5,12-14]$. To our knowledge, this study is the first to use global expression profiling to characterize the human skin transcriptome during early $B$ burgdorferi infection. Our findings are particularly relevant to patients with evidence of dissemination of $B$ burgdorferi to the blood and/or to other skin sites.

Detection by pattern recognition molecules (PRMs) of the innate immune response is the first line of defense against invading microbes. Our data show upregulation of TLR1 and $T L R 2$, which are known to be necessary for optimal recognition of the spirochete by phagocytes $[15,16]$. Also upregulated were PTX3 (pentraxin 3), C1QB (complement component 1, q subcomponent, B chain), and FCN1 (ficolin-1), which are part of the humoral arm of innate immunity [17]. Pentraxin 3 binds the complement component $\mathrm{C} 1 \mathrm{q}$, influences complement activation, and has opsonic activity [18]. Ficolin-1 is also involved in opsonophagocytosis and complement activation via the lectin pathway, and it interacts with PTX3 [19]. The role of these PRMs in Lyme disease is unknown at this point. Also upregulated was CLEC7A (dectin-1), a C-type lectin receptor that recognizes $\beta$-glucans. This is interesting, because a study investigating dectin-1 in B burgdorferi infection in mice found no major role for this receptor [20].

Monocytes/macrophages and neutrophils are among the first innate cells encountered during early infection. Both cell types are able to phagocytose $B$ burgdorferi [21], and mononuclear phagocytes are important in reducing the number of spirochetes in tissues [22]. Our data reveal that many components involved in phagocytosis of $B$ burgdorferi are induced in the EM skin lesion, including genes important for the recognition of the spirochete as a phagocytic target (TLR2, TLR1, CR3, FCGR1A, FCGR1B, FCGR3A/FCGR3B). Also upregulated was CYBB (cytochrome b-245, beta polypeptide), encoding the catalytic subunit gp91 $91^{\text {phox }}$ of the superoxide-generating NADPH oxidase, which is considered essential for killing of phagocytosed pathogens [23].

The most upregulated genes in our dataset were those encoding CXCL9, CXCL10, and CXCL11. These chemokines are ligands for the CXCR3 receptor, expressed in $\mathrm{CD}^{+}$ type-1 helper (Th1) and $\mathrm{CD}^{+}$cytotoxic lymphocytes, and are 
A

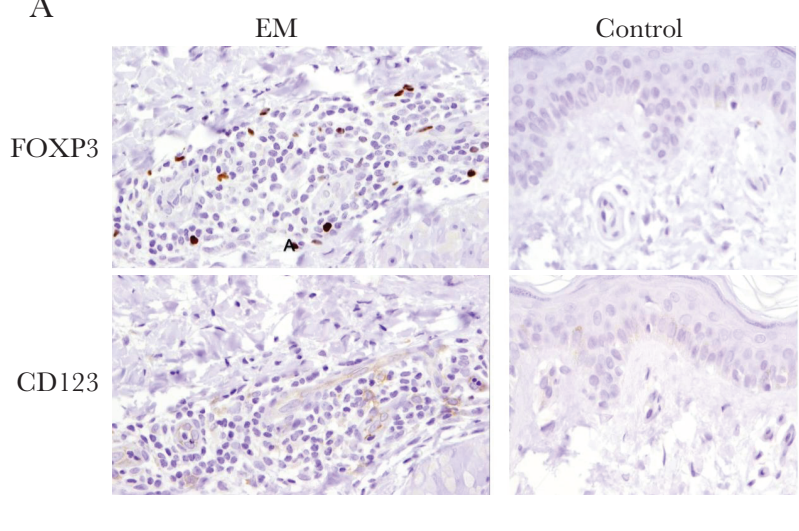

B

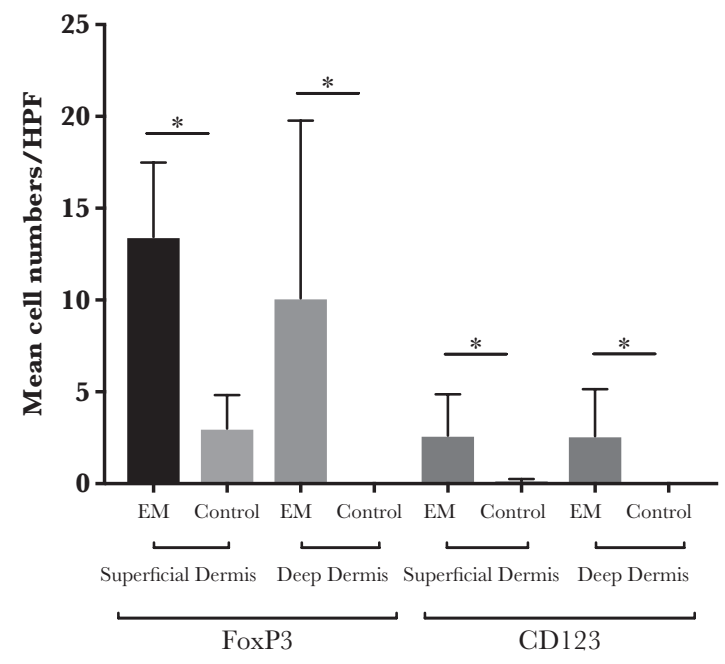

Figure 4. Immunohistochemical identification of immune cell types present in erythema migrans (EM) skin lesions. (A) Thin sections of paraffin-embedded skin biopsies from an EM lesion (left panel) and a surgical control (right panel) were incubated with antibodies specific for forkhead box P3 (FoxP3), a marker of T regulatory cells, and the interleukin-3 receptor $\alpha$ chain (CD123), a marker of plasmacytoid dendritic cells. Photos were taken at $\times 60$ magnification. $(B)$ The mean cell counts per high-power field for cells expressing FoxP3 and CD123 were significantly different when comparing EM lesions (8 patients) with control skin (2 skin specimens from a healthy volunteer and 2 surgical controls). ${ }^{*}$, 2-tailed $P$ value $<.05$, MannWhitney test. Error bars show standard deviation.

important for migration of these cells into inflammatory sites [24]. CXCL9 and CXCL10 induce polarization of CD4 ${ }^{+} \mathrm{T}$ cells into effector Th1/Th17 cells, whereas CXCL11 drives T-cell polarization into $T$ regulatory 1 cell subset [25]. Multiple studies have documented a correlation between elevated expression of these chemokines and Lyme disease pathogenesis [12, 26-28]; and levels of CXCL9, CXCL10, and CXCL11 were higher in EM caused by $B$ burgdorferi compared with Borreliella afzelii [14].

The transcriptional profile in EM biopsies showed a dominant IFN signature, strongly characterized by the expression of an array of IFN-responsive genes. Although pathway analysis predicted both IFN- $\gamma$ and IFN- $\alpha$ as upstream regulators, we found only IFNG to be increased in the EM biopsy samples, both by microarray and by RT-PCR. The presence of IFN- $\gamma$ in EM lesions has been well established. High levels of IFN- $\gamma$ were detected in the blister fluids raised over EM lesions [5]. In another study, patients who had lower levels of IFN- $\gamma$ in the EM skin biopsy were more likely to report persisting symptoms at 6 months posttreatment [13], suggesting that a strong IFN- $\gamma$ response at the site of spirochete inoculation has host-protective effects.

Notably, we found that EM lesions are associated with a significant increase in the gene expression of 3 enzymes controlling tryptophan catabolism: indoleamine 2,3-dioxygenase 1 (IDO1), kynureninase (KYNU), and kynurenine 3-monooxygenase $(K M O)$. Indoleamine 2,3-dioxygenase 1 is predominantly expressed by antigen-presenting cells and plays a pivotal role in balancing the immune response during infections caused by a variety of pathogens, including bacteria [29-32]. Indoleamine 2,3-dioxygenase 1 is the first and rate-limiting enzyme in the degradation of tryptophan, leading to diminished tryptophan levels and increased kynurenine pathway metabolites. The depletion of tryptophan in the local microenvironment may have antimicrobial effects (B burgdorferi is a tryptophan auxotroph), but it also leads to inhibition of T-cell responses, resulting in local immunosuppression and tolerance [33]. Of particular relevance to Lyme disease, IDO1 may promote pathogen invasion of epithelial tissues through the generation of localized immunosuppression [34]. We previously observed that $B$ burgdorferi strains more frequently associated with disseminated infection induced significantly higher levels of IFN- $\alpha$ in human peripheral blood mononuclear cells [35] and that both type I and type II IFN signaling are required for $B$ burgdorferi to elicit maximal production of IDO1 in this system [36]. The presence of Tregs and plasmacytoid DCs in the inflammatory infiltrate of EM lesions adds support to a possible role of an IDO1-mediated tolerogenic mechanism in the host response to $B$ burgdorferi.

Collectively, our data delineate host defense strategies to detect and eliminate B burgdorferi, as well as tolerance mechanisms that promote localized immunosuppression at the site of inoculation, which may be exploited by the pathogen, and allow for disease progression. A proposed model based on our results is presented in Figure 5. Stimulation of dermal fibroblasts by migrating spirochetes leads to the production of proinflammatory chemokines including CCL2, CXCL1, and CXCL10 [37]. The heterodimer S100A8/A9 (calprotectin), expressed by keratinocytes, granulocytes, monocytes, and DCs, exerts antimicrobial effects against bacteria, including $B$ burgdorferi, via metal sequestration [38], but may also have chemotactic effects [39]. Macrophages and DCs are recruited to the infection site, where TLR-mediated recognition of $B$ burgdorferi results in nuclear factor- $\mathrm{kB}$-dependent cytokine and chemokine production. CXCL9, CXCL10, and CXCL11 attract leukocytes to the infected sites where CXCL9 and CXCL10 promote the polarization of $\mathrm{CD}^{+} \mathrm{T}$ cells into effector Th1/Th17 cells. The Th1 cells produce IFN- $\gamma$, enhancing the phagocytic activity of macrophages. 


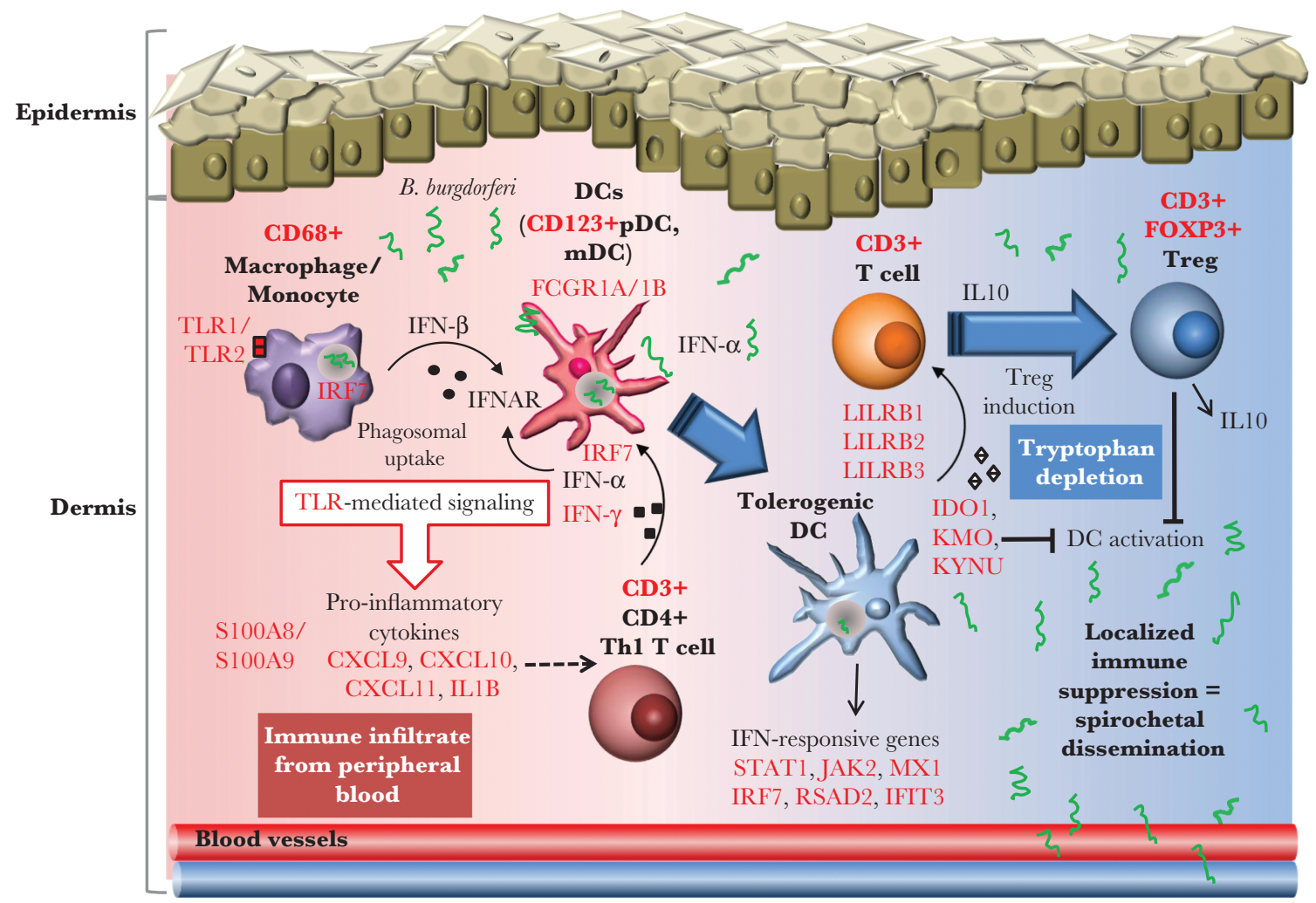

Figure 5. Proposed model of immunopathogenesis of Borreliella burgdorferi during early cutaneous infection. After deposition into the skin by the tick vector, $B$ burgdorferi are detected by Toll-like receptors (TLRs) of resident innate immune cells, notably tissue macrophages (histiocytes) and dendritic cells (DCs), resulting in the expression of inflammatory cytokines that recruit leukocytes from the peripheral blood and drive the differentiation of interferon (IFN)- $\gamma$-expressing Th1 cells. Calprotectin, an antimicrobial heterodimer (S100A8/S100A9) secreted by keratinocytes, mononuclear cells, and granulocytes may contribute to immune cell recruitment. Some spirochetes are internalized by phagocytic immune cells, possibly via Fc gamma receptors (FCGR1A, FCGR1B), and elicit production of type I IFNs through endosomal TLRs and the IRF7 signaling pathway. Interferon- $\alpha$ and IFN- $\gamma$ stimulate the expression of IFN-responsive genes through JAK/STAT signaling and, in combination, shape the development of ID01-expressing tolerogenic DCs that drive the differentiation of regulatory T cells through tryptophan depletion. Borreliella burgdorferi may exploit the resulting localized immunosuppression to promote hematogenous dissemination to distal tissues. Symbols for genes with significantly elevated expression in EM biopsies relative to control skin are indicated in red font.

Phagosomal TLR signaling by DCs results in production of type I IFNs that, in combination with IFN- $\gamma$, induce IFN-responsive genes, including those encoding key enzymes in the tryptophan catabolic pathway (IDO1, KMO, KYNU) [40]. Tryptophan depletion suppresses $\mathrm{CD} 8^{+} \mathrm{T}$-cell priming and drives the development of FoxP3 ${ }^{+}$Treg cells [33] through increased expression of immunoglobulin-like receptors (LILRB1, LILRB2, LILRB3) [41], consequently generating a region of localized immune suppression that may facilitate spirochetal dissemination.

\section{CONCLUSIONS}

In conclusion, we demonstrate that EM lesions exhibit an IFNassociated transcriptomic signature that features important regulatory factors involved in host defense as well as in immune modulation. The capacity of $B$ burgdorferi to manipulate and exploit these pathways may play a pivotal role in determining the outcome of infection. Our data provide new insights into the immunopathogenesis of early cutaneous Lyme disease and uncover multiple targets for further research.

\section{Data Availability Statement}

The dataset for this article is publicly available in the GEO database at GSE154916.

\section{Supplementary Data}

Supplementary materials are available at The Journal of Infectious Diseases online. Consisting of data provided by the authors to benefit the reader, the posted materials are not copyedited and are the sole responsibility of the authors, so questions or comments should be addressed to the corresponding author.

\section{Notes}

Acknowledgments. We thank Zoila Rangel for the help with the microarray data analysis. We also thank the staff of the Division of Infectious Diseases of New York Medical College for their assistance in performing the erythema migrans skin biopsies and Dr. Praveen Sudhindra for assistance during the study.

Disclaimer. The funding sources had no role in study design, interpretation and in the writing of the manuscript. The 
content of this publication does not necessarily reflect the views of, or policies of the Department of Health and Human Services, nor does mention of trade names, commercial products, or organizations imply endorsement by the US Government.

Financial support. This work was supported by the Intramural Research Program of the National Institutes of Health, National Institute of Allergy and Infectious Diseases. This project has been funded in whole or in part with federal funds from the National Cancer Institute, National Institutes of Health, under Contract No. HHSN261200800001E; the United States Centers for Disease Control and Prevention (grant numbers U01CI000160 [to I. S.] and U01CK000153 [to I. S. and M. M. P.]); and the National Institutes of Health (grant AI45801 to I. S.).

Potential conflicts of interest. All authors: No reported conflicts of interest. All authors have submitted the ICMJE Form for Disclosure of Potential Conflicts of Interest.

\section{References}

1. Mead PS. Epidemiology of Lyme disease. Infect Dis Clin North Am 2015; 29:187-210.

2. Steere AC. Lyme disease. N Engl J Med 2001; 345:115-25.

3. Böer A, Bresch M, Dayrit J, Falk TM. Erythema migrans: a reassessment of diagnostic criteria for early cutaneous manifestations of borreliosis with particular emphasis on clonality investigations. Br J Dermatol 2007; 156:1263-71.

4. Müllegger RR, McHugh G, Ruthazer R, Binder B, Kerl H, Steere AC. Differential expression of cytokine mRNA in skin specimens from patients with erythema migrans or acrodermatitis chronica atrophicans. J Invest Dermatol 2000; 115:1115-23.

5. Salazar JC, Pope CD, Sellati TJ, et al. Coevolution of markers of innate and adaptive immunity in skin and peripheral blood of patients with erythema migrans. J Immunol 2003; 171:2660-70.

6. Centers for Disease and Control and Prevention. Lyme Disease (Borrelia burgdorferi) 2017 Case Definition. Available at: http://wwwn.cdc.gov/NNDSS/conditions/lyme-disease/ case-definition/2017/. Accessed 8 November 2017.

7. Wormser GP, Liveris D, Nowakowski J, et al. Association of specific subtypes of Borrelia burgdorferi with hematogenous dissemination in early Lyme disease. J Infect Dis 1999; 180:720-5.

8. Schwartz I, Wormser GP, Schwartz JJ, et al. Diagnosis of early Lyme disease by polymerase chain reaction amplification and culture of skin biopsies from erythema migrans lesions. J Clin Microbiol 1992; 30:3082-8.

9. Jiao X, Sherman BT, Huang da W, et al. DAVID-WS: a stateful web service to facilitate gene/protein list analysis. Bioinformatics 2012; 28:1805-6.

10. Rusinova I, Forster S, Yu S, et al. Interferome v2.0: an updated database of annotated interferon-regulated genes. Nucleic Acids Res 2013; 41:D1040-6.
11. Fallarino F, Grohmann U, Puccetti P. Indoleamine 2, 3-dioxygenase: from catalyst to signaling function. Eur J Immunol 2012; 42:1932-7.

12. Müllegger RR, Means TK, Shin JJ, et al. Chemokine signatures in the skin disorders of Lyme borreliosis in Europe: predominance of CXCL9 and CXCL10 in erythema migrans and acrodermatitis and CXCL13 in lymphocytoma. Infect Immun 2007; 75:4621-8.

13. Sjöwall J, Fryland L, Nordberg M, et al. Decreased Th1-type inflammatory cytokine expression in the skin is associated with persisting symptoms after treatment of erythema migrans. PLoS One 2011; 6:e18220.

14. Jones KL, Muellegger RR, Means TK, et al. Higher mRNA levels of chemokines and cytokines associated with macrophage activation in erythema migrans skin lesions in patients from the United States than in patients from Austria with Lyme borreliosis. Clin Infect Dis 2008; 46:85-92.

15. Hirschfeld M, Kirschning CJ, Schwandner R, et al. Cutting edge: inflammatory signaling by Borrelia burgdorferi lipoproteins is mediated by Toll-like receptor 2. J Immunol 1999; 163:2382-6.

16. Sellati TJ, Sahay B, Wormser GP. The Toll of a TLR1 polymorphism in Lyme disease: a tale of mice and men. Arthritis Rheum 2012; 64:1311-5.

17. Garlanda C, Jaillon S, Doni A, Bottazzi B, Mantovani A. PTX3, a humoral pattern recognition molecule at the interface between microbe and matrix recognition. Curr Opin Immunol 2016; 38:39-44.

18. Bottazzi $B$, Inforzato $A$, Messa $M$, et al. The pentraxins PTX3 and SAP in innate immunity, regulation of inflammation and tissue remodelling. J Hepatol 2016; 64:1416-27.

19. Doni A, Garlanda C, Bottazzi B, Meri S, Garred P, Mantovani A. Interactions of the humoral pattern recognition molecule PTX3 with the complement system. Immunobiology 2012; 217:1122-8.

20. Oosting M, Buffen K, Cheng SC, et al. Borrelia-induced cytokine production is mediated by spleen tyrosine kinase (Syk) but is Dectin-1 and Dectin-2 independent. Cytokine 2015; 76:465-72.

21. Benach JL, Fleit HB, Habicht GS, Coleman JL, Bosler EM, Lane BP. Interactions of phagocytes with the Lyme disease spirochete: role of the Fc receptor. J Infect Dis 1984; 150:497-507.

22. Carrasco SE, Troxell B, Yang Y, et al. Outer surface protein OspC is an antiphagocytic factor that protects Borrelia burgdorferi from phagocytosis by macrophages. Infect Immun 2015; 83:4848-60.

23. Lambeth JD. NOX enzymes and the biology of reactive oxygen. Nat Rev Immunol 2004; 4:181-9.

24. Groom JR, Luster AD. CXCR3 in T cell function. Exp Cell Res 2011; 317:620-31.

25. Karin N, Wildbaum G. The role of chemokines in adjusting the balance between CD4+ effector $\mathrm{T}$ cell subsets and 
FOXp3-negative regulatory T cells. Int Immunopharmacol 2015; 28:829-35.

26. Lepej SZ, Rode OD, Jeren T, Vince A, Remenar A, Barsić B. Increased expression of CXCR3 and CCR5 on memory CD4+ T-cells migrating into the cerebrospinal fluid of patients with neuroborreliosis: the role of CXCL10 and CXCL11. J Neuroimmunol 2005; 163:128-34.

27. Shin JJ, Glickstein LJ, Steere AC. High levels of inflammatory chemokines and cytokines in joint fluid and synovial tissue throughout the course of antibiotic-refractory lyme arthritis. Arthritis Rheum 2007; 56:1325-35.

28. Soloski MJ, Crowder LA, Lahey LJ, Wagner CA, Robinson WH, Aucott JN. Serum inflammatory mediators as markers of human Lyme disease activity. PLoS One 2014; 9:e93243.

29. de Souza Sales J, Lara FA, Amadeu TP, et al. The role of indoleamine 2, 3-dioxygenase in lepromatous leprosy immunosuppression. Clin Exp Immunol 2011; 165:251-63.

30. Li W, Katz BP, Spinola SM. Haemophilus ducreyi lipooligosaccharides induce expression of the immunosuppressive enzyme indoleamine 2, 3-dioxygenase via type I interferons and tumor necrosis factor alpha in human dendritic cells. Infect Immun 2011; 79:3338-47.

31. Loughman JA, Hunstad DA. Induction of indoleamine 2, 3-dioxygenase by uropathogenic bacteria attenuates innate responses to epithelial infection. J Infect Dis 2012; 205:1830-9.

32. Popov A, Abdullah Z, Wickenhauser C, et al. Indoleamine 2, 3-dioxygenase-expressing dendritic cells form suppurative granulomas following Listeria monocytogenes infection. J Clin Invest 2006; 116:3160-70.

33. Barth H, Raghuraman S. Persistent infectious diseases say IDO. Role of indoleamine-2, 3-dioxygenase in disease pathogenesis and implications for therapy. Crit Rev Microbiol 2014; 40:360-8.
34. Scheler M, Wenzel J, Tüting T, Takikawa O, Bieber T, von Bubnoff D. Indoleamine 2, 3-dioxygenase (IDO): the antagonist of type I interferon-driven skin inflammation? Am J Pathol 2007; 171:1936-43.

35. Love AC, Schwartz I, Petzke MM. Borrelia burgdorferi RNA induces type I and III interferons via Toll-like receptor 7 and contributes to production of NF- $\mathrm{BB}$-dependent cytokines. Infect Immun 2014; 82:2405-16.

36. Love AC, Schwartz I, Petzke MM. Induction of indoleamine 2, 3-dioxygenase by Borrelia burgdorferi in human immune cells correlates with pathogenic potential. J Leukoc Biol 2015; 97:379-90.

37. Meddeb M, Carpentier W, Cagnard N, et al. Homogeneous inflammatory gene profiles induced in human dermal fibroblasts in response to the three main species of Borrelia burgdorferi sensu lato. PLoS One 2016; 11:e0164117.

38. Lusitani D, Malawista SE, Montgomery RR. Borrelia burgdorferi are susceptible to killing by a variety of human polymorphonuclear leukocyte components. J Infect Dis 2002; 185:797-804.

39. Lee Y, Jang S, Min JK, et al. S100A8 and S100A9 are messengers in the crosstalk between epidermis and dermis modulating a psoriatic milieu in human skin. Biochem Biophys Res Commun 2012; 423:647-53.

40. Petzke MM, Brooks A, Krupna MA, Mordue D, Schwartz I. Recognition of Borrelia burgdorferi, the Lyme disease spirochete, by TLR7 and TLR9 induces a type I IFN response by human immune cells. J Immunol 2009; 183:5279-92.

41. Brenk M, Scheler M, Koch S, et al. Tryptophan deprivation induces inhibitory receptors ILT3 and ILT4 on dendritic cells favoring the induction of human CD4+CD25+ Foxp3+ T regulatory cells. J Immunol 2009; 183:145-54. 